\title{
A MONOTONE PROCESS MAINTENANCE MODEL FOR A MULTISTATE SYSTEM
}

\author{
LAM YEH, ${ }^{*}$ Northeastern University at Qinhuangdao and University of Hong Kong
}

\begin{abstract}
In this paper, we study a monotone process maintenance model for a multistate system with $k$ working states and $\ell$ failure states. By making different assumptions, we can apply the model to a multistate deteriorating system as well as to a multistate improving system. We show that the monotone process model for a multistate system is equivalent to a geometric process model for a two-state system. Then, for both the deteriorating and the improving system, we analytically determine an optimal replacement policy for minimizing the long-run average cost per unit time.
\end{abstract}

Keywords: Geometric process; replacement policy $N$; harmonic mean; renewal reward process

2000 Mathematics Subject Classification: Primary 90B25

Secondary 60K 10

\section{Introduction}

In practice, because of ageing effects and accumulated wearing, many systems deteriorate in such a way that the successive operating times become shorter and shorter, while the repair times become longer and longer. Therefore, the use of a monotone process model is a direct approach to the maintenance problem of a deteriorating system. To introduce such a model, we first introduce the concept of stochastic order.

Definition 1. A random variable $X$ is said to be stochastically greater or less than a random variable $Y$ if, respectively, $\mathrm{P}(X>\alpha) \geq \mathrm{P}(Y>\alpha)$ or $\mathrm{P}(X>\alpha) \leq \mathrm{P}(Y>\alpha)$ for all real $\alpha$. This ordering is denoted by $X \geq_{\text {st }} Y$ or $X \leq_{\text {st }} Y$, as appropriate (see Ross (1996)). Furthermore, a stochastic process $\left\{X_{n}, n=1,2, \ldots\right\}$ is said to be stochastically increasing or decreasing if $X_{n+1} \geq_{\text {st }} X_{n}$ or $X_{n+1} \leq_{\text {st }} X_{n}$, respectively, for $n=1,2, \ldots$

Note that the stochastic order defined by Definition 1 is the usual one. However, the stochastically monotone concept for a stochastic process $\left\{X_{n}, n=1,2, \ldots\right\}$ defined here is simply based on the distributions of $X_{n}$ and $X_{n+1}$ for $n=1,2, \ldots$ This is different from the stochastically monotone concept for a Markov process. Recall that a Markov process $\left\{X_{n}, n=1,2, \ldots\right\}$ with state space $\{0,1, \ldots\}$ is said to be stochastically monotone if

$$
\left(X_{n+1} \mid X_{n}=i_{1}\right) \leq_{\mathrm{st}}\left(X_{n+1} \mid X_{n}=i_{2}\right)
$$

for any $0 \leq i_{1} \leq i_{2}$ (see, e.g. Shaked and Shanthikumar (1994)). Clearly, the stochastically monotone concept for a Markov process is defined for a Markov process and is based on the probabilities of transition from one state to another state, conditioning on the former state.

Received 19 May 2003; revision received 27 August 2004.

* Postal address: Department of Statistics and Actuarial Science, University of Hong Kong, Pokfulam Road, Hong Kong. Email address: ylam@hkustasc.hku.hk 
However, the stochastically monotone concept for a stochastic process defined here is for a general process, and is based on the unconditional distributions of two successive random variables in the process.

Lam (1988a), (1988b) introduced the geometric process as a simple monotone process.

Definition 2. A stochastic process $\left\{X_{n}, n=1,2, \ldots\right\}$ is a geometric process (GP), if there exists a real $a>0$ such that $\left\{a^{n-1} X_{n}, n=1,2, \ldots\right\}$ forms a renewal process. The number $a$ is called the ratio of the GP.

Obviously, if $0<a \leq 1$, the GP is stochastically increasing; if $a \geq 1$, it is stochastically decreasing; and if $a=1$, the GP will reduce to a renewal process.

The GP was applied to the maintenance problem of a one-component system by Lam (1988a), (1988b), (2003). Thereafter, the GP was also used in the study of the maintenance problem for a two-component system; see Lam (1995), Lam and Zhang (1996), and Zhang (1999). Furthermore, Lam (1992a), Lam and Chan (1998), and Lam et al. (2004) applied the GP model to the analysis of data from a series of events. For further reference, see Leung and Lee (1998), Sheu (1999), and Pérez-Ocón and Torres-Castro (2002). Moreover, see Lam (2005) for a brief review and more references.

However, most of the research work has so far considered a two-state system only, with up and down states, say. Little attention has been paid to the study of an improving system. In many practical situations, a system may have more than two states. For example, the system constituted by an electronic instrument may break down due to a short circuit or an open circuit, in which case the system will have two failure states and one working state. Moreover, although many practical systems are deteriorating, there do exist some improving systems. For example, the successive operating times of a system after repair might be prolonged due to the system operator's accumulation of working experience and/or the replacement, during repair, of some failed parts of the system by more advanced parts. Consecutive repair times after failure might, furthermore, be shortened because the repair facility becomes more and more familiar with the system. Therefore, it is necessary to study a maintenance model for both a multistate deteriorating system and a multistate improving system. Lam et al. (2002) considered a monotone process model for a multistate deteriorating system with $k$ failure states and one working state. Zhang et al. (2002) also studied a monotone process model for the maintenance problem of a multistate system.

In this paper, we will study a monotone process model for a one-component multistate system with $k+\ell$ states, namely $k$ working states and $\ell$ failure states. By making different assumptions, the model can be applied to a multistate deteriorating system as well as to a multistate improving system. The replacement policy $N$ is applied, by which the system will be replaced following the $N$ th failure. Then, we shall show that the monotone process model for the multistate system is equivalent to a GP model for a two-state system in the sense that two systems will have the same long-run average cost per unit time and the same optimal replacement policy. Finally, the optimal replacement policy is determined analytically for both the deteriorating system and the improving system.

\section{The monotone process model}

Suppose that we are given a one-component multistate system. We will first describe the probability structure of the model. To do this, we note that the system state at time $t$ can be 
denoted by

$$
S(t)= \begin{cases}i & \text { if the system is in the } i \text { th working state at time } t(i=1, \ldots, k), \\ k+i & \text { if the system is in the } i \text { th failure state at time } t(i=1, \ldots, \ell)\end{cases}
$$

The set of working states is $W=\{1, \ldots, k\}$, the set of failure states is $F=\{k+1, \ldots, k+\ell\}$, and the state space is $\Omega=W \cup F$. Initially, assume that a new system in working state 1 is installed. Whenever the system fails, it will be repaired. Let $t_{n}$ be the completion time of the $n$th repair, $n=0,1, \ldots$, with $t_{0}=0$, and let $s_{n}$ be the time of the $n$th failure, $n=1,2, \ldots$ Then, clearly we have

$$
t_{0}<s_{1}<t_{1}<\cdots<s_{n}<t_{n}<\cdots .
$$

Furthermore, assume that the transition probability from working state $i, i=1, \ldots, k$, to failure state $k+j, j=1, \ldots, \ell$, is given by

$$
\mathrm{P}\left(S\left(s_{n+1}\right)=k+j \mid S\left(t_{n}\right)=i\right)=q_{j},
$$

with $\sum_{j=1}^{\ell} q_{j}=1$. Moreover, the transition probability from failure state $k+i, i=1, \ldots, \ell$, to working state $j, j=1, \ldots, k$, is given by

$$
\mathrm{P}\left(S\left(t_{n}\right)=j \mid S\left(s_{n}\right)=k+i\right)=p_{j},
$$

with $\sum_{j=1}^{k} p_{j}=1$.

Let $X_{1}$ be the operating time of a system after installation. In general, let $X_{n}, n=2,3, \ldots$, be the operating time of the system after the $(n-1)$ th repair and let $Y_{n}, n=1,2, \ldots$, be the repair time after the $n$th failure. Assume that there exist a lifetime distribution $U(t)$ and an $a_{i}>0, i=1, \ldots, k$, such that

$$
\mathrm{P}\left(X_{1} \leq t\right)=U(t)
$$

and

$$
\mathrm{P}\left(X_{2} \leq t \mid S\left(t_{1}\right)=i\right)=U\left(a_{i} t\right), \quad i=1, \ldots, k
$$

In general,

$$
\begin{aligned}
\mathrm{P}\left(X_{n} \leq t \mid S\left(t_{1}\right)=i_{1}, \ldots,\right. & \left.S\left(t_{n-1}\right)=i_{n-1}\right) \\
= & U\left(a_{i_{1}} \ldots a_{i_{n-1}} t\right), \quad i_{j}=1, \ldots, k, j=1, \ldots, n-1 .
\end{aligned}
$$

Similarly, there exist a lifetime distribution $V(t)$ and a $b_{i}>0, i=1, \ldots, \ell$, such that

$$
\mathrm{P}\left(Y_{1} \leq t \mid S\left(s_{1}\right)=k+i\right)=V\left(b_{i} t\right)
$$

and, in general,

$$
\begin{aligned}
\mathrm{P}\left(Y_{n} \leq t \mid S\left(s_{1}\right)=k+i_{1}, \ldots,\right. & \left.S\left(s_{n}\right)=k+i_{n}\right) \\
& =V\left(b_{i_{1}} \ldots b_{i_{n}} t\right), \quad i_{j}=1, \ldots, \ell, j=1, \ldots, n .
\end{aligned}
$$

We now make additional assumptions on the policy used, the cost structure, and the monotonicity conditions of the model.

Assumption 1. A replacement policy $N$ is applied, by which the system is replaced by a new (identical) one following the N th failure. 
Assumption 2. If a system in working state $i$ is operating, the reward rate is $r_{i}, i=1, \ldots, k$. If the system in failure state $k+i$ is under repair, the repair-cost rate is $c_{i}, i=1, \ldots, \ell$. The replacement cost comprises two parts: one part is the basic replacement cost $R$ and the other is proportional to the replacement time $Z$ at rate $c_{p}$. In other words, the replacement cost is given by $R+c_{p} Z$.

Assumption 3. We assume that

$$
\begin{aligned}
& 1=a_{1} \leq a_{2} \leq \cdots \leq a_{k}, \\
& 1=b_{1} \geq b_{2} \geq \cdots \geq b_{\ell}>0 .
\end{aligned}
$$

Under the additional Assumptions 1-3, we shall argue that the model is a maintenance model for a multistate deteriorating system. For a multistate improving system, Assumption 3 will be replaced by the following assumption.

Assumption $3^{\prime}$. We assume that

$$
\begin{aligned}
& 1=a_{1} \geq a_{2} \geq \cdots \geq a_{k}>0, \\
& 1=b_{1} \leq b_{2} \leq \cdots \leq b_{\ell} .
\end{aligned}
$$

Note that the equalities in (5) and (6) do not all hold simultaneously.

Remarks. The use of policy $N$ has a long history (see, e.g. Morimura (1970) and Park (1979)). However, in maintenance problems, replacement policy $T$ - by which the system is replaced at a stopping time $T$ - is also used, where $T$ is the total operating time. It is interesting to compare policies $N$ and $T$. In studying the long-run average cost per unit time, Stadje and Zuckerman (1990) and Lam (1991b) showed that, under some mild conditions, an optimal policy $N^{*}$ is at least as good as an optimal policy $T^{*}$. In the case of the total expected discounted cost, the same result was proven by Lam (1991a), (1992b). The implementation of a policy $N$ is also much simpler than that of a policy $T$. These are the reasons we are using policy $N$ here.

Since the system has $k$ different working states, it follows that in each one it should have a different reward rate. Similarly, in different failure states, the system should have different repair-cost rates.

Under Assumption 3, for two working states $0 \leq i_{1}<i_{2} \leq k$, we have

$$
\left(X_{2} \mid S\left(t_{1}\right)=i_{1}\right) \geq_{\mathrm{st}}\left(X_{2} \mid S\left(t_{1}\right)=i_{2}\right) .
$$

Therefore, working state $i_{1}$ is better than working state $i_{2}$ in the sense that the system in state $i_{1}$ has a stochastically larger operating time than it does in state $i_{2}$. Consequently, the $k$ working states are arranged in decreasing order, such that state 1 is the best working state and state $k$ is the worst working state. Similarly, for two failure states $k+i_{1}$ and $k+i_{2}$ such that $k+1 \leq k+i_{1}<k+i_{2} \leq k+\ell$,

$$
\left(Y_{1} \mid S\left(s_{1}\right)=k+i_{1}\right) \leq_{\mathrm{st}}\left(Y_{1} \mid S\left(s_{1}\right)=k+i_{2}\right) .
$$

Therefore, failure state $k+i_{1}$ is better than failure state $k+i_{2}$ in the sense that the system in state $k+i_{1}$ has a stochastically smaller repair time than it does in state $k+i_{2}$. Thus, the $\ell$ failure states are also arranged in decreasing order, such that state $k+1$ is the best failure state and state $k+\ell$ is the worst failure state. 
In particular, if $p_{1}=q_{1}=0, a_{2}=\cdots=a_{k}=a$, and $b_{2}=\cdots=b_{\ell}=b$, then the system reduces to a two-state system. In fact, (1) and (2) now become $\mathrm{P}\left(X_{n} \leq t\right)=U\left(a^{n-1} t\right)$ and $\mathrm{P}\left(Y_{n} \leq t\right)=V\left(b^{n} t\right)$, respectively. Thus, $\left\{X_{n}, n=1,2, \ldots\right\}$ will form a GP with ratio $a \geq 1$ and $X_{1} \sim U$, while $\left\{Y_{n}, n=1,2, \ldots\right\}$ will form a GP with ratio $0<b \leq 1$ and $Y_{1} \sim G$ where $G(t)=V(b t)$. As a result, our model reduces to the GP model for the one-component two-state system introduced by Lam (1988a), (1988b).

Under Assumption 3', for two working states $0 \leq i_{1}<i_{2} \leq k$ a similar argument shows that working state $i_{1}$ is worse than working state $i_{2}$ since the system in state $i_{1}$ has a stochastically smaller operating time than it does in state $i_{2}$. This means that the $k$ working states are arranged in increasing order, such that state 1 is the worst working state and state $k$ is the best working state. On the other hand, for two failure states $k+1 \leq k+i_{1}<k+i_{2} \leq k+\ell$, failure state $k+i_{1}$ is worse than failure state $k+i_{2}$ since the system in state $k+i_{1}$ has a stochastically larger repair time than it does in state $k+i_{2}$. Therefore, the $\ell$ failure states are also arranged in increasing order, such that state $k+1$ is the worst failure state and state $k+\ell$ is the best failure state. Note that if all the equalities in (5) and (6) hold, this will be a special case of (3) and (4). In this case, Assumption 3 will hold and it should be excluded from Assumption 3'.

Now we shall demonstrate that our model is a monotone process model for the multistate system. To start with, we first observe that

$$
\mathrm{P}\left(X_{2} \leq t\right)=\sum_{i=0}^{k-1} \mathrm{P}\left(X_{2} \leq t \mid S\left(t_{1}\right)=i\right) \mathrm{P}\left(S\left(t_{1}\right)=i\right)=\sum_{i=1}^{k} p_{i} U\left(a_{i} t\right) .
$$

In general,

$$
\begin{aligned}
\mathrm{P}\left(X_{n} \leq t\right)= & \sum_{i_{1}=1}^{k} \ldots \sum_{i_{n-1}=1}^{k} \mathrm{P}\left(X_{n} \leq t \mid S\left(t_{1}\right)=i_{1}, \ldots, S\left(t_{n-1}\right)=i_{n-1}\right) \\
& \times \mathrm{P}\left(S\left(t_{1}\right)=i_{1}, \ldots, S\left(t_{n-1}\right)=i_{n-1}\right) \\
= & \sum_{i_{1}=1}^{k} \cdots \sum_{i_{n-1}=1}^{k} p_{i_{1}} \cdots p_{i_{n-1}} U\left(a_{i_{1}} \cdots a_{i_{n-1}} t\right) \\
= & \sum_{\left\{j_{1}, \ldots, j_{k} \in \mathbb{Z}^{+}: \sum_{i=1}^{k} j_{i}=n-1\right\}} \frac{(n-1) !}{j_{1} ! \cdots j_{k} !} p_{1}^{j_{1}} \cdots p_{k}^{j_{k}} U\left(a_{1}^{j_{1}} \cdots a_{k}^{j_{k}} t\right)
\end{aligned}
$$

and, similarly,

$$
\begin{aligned}
\mathrm{P}\left(Y_{n} \leq t\right)= & \sum_{i_{1}=1}^{\ell} \cdots \sum_{i_{n}=1}^{\ell} \mathrm{P}\left(Y_{n} \leq t \mid S\left(s_{1}\right)=k+i_{1}, \ldots, S\left(s_{n}\right)=k+i_{n}\right) \\
& \times \mathrm{P}\left(S\left(s_{1}\right)=k+i_{1}, \ldots, S\left(s_{n}\right)=k+i_{n}\right) \\
= & \sum_{i_{1}=1}^{\ell} \cdots \sum_{i_{n}=1}^{\ell} q_{i_{1}} \cdots q_{i_{n}} V\left(b_{i_{1}} \cdots b_{i_{n}} t\right) \\
= & \sum_{\left\{j_{1}, \ldots, j_{l} \in \mathbb{Z}^{+}: \sum_{i=1}^{l} j_{i}=n\right\}} \frac{n !}{j_{1} ! \cdots j_{\ell} !} q_{1}^{j_{1}} \cdots q_{\ell}^{j_{\ell}} V\left(b_{1}^{j_{1}} \cdots b_{\ell}^{j_{\ell}} t\right) .
\end{aligned}
$$


Second, we will show that, under the additional Assumption $3,\left\{X_{n}, n=1,2, \ldots\right\}$ is stochastically decreasing while $\left\{Y_{n}, n=1,2, \ldots\right\}$ is stochastically increasing. This is the following theorem.

Theorem 1. Under Assumption 3, for $n=1,2, \ldots$, we have $X_{n} \geq_{\mathrm{st}} X_{n+1}$ and $Y_{n} \leq_{\mathrm{st}} Y_{n+1}$.

Proof. For any $t>0$, it follows from (7) that

$$
\begin{aligned}
\mathrm{P}\left(X_{n+1} \leq t\right) & =\sum_{i_{1}=1}^{k} \cdots \sum_{i_{n}=1}^{k} p_{i_{1}} \cdots p_{i_{n}} U\left(a_{i_{1}} \cdots a_{i_{n}} t\right) \\
& =\sum_{i_{1}=1}^{k} \cdots \sum_{i_{n-1}=1}^{k} p_{i_{1}} \cdots p_{i_{n-1}}\left[\sum_{i_{n}=1}^{k} p_{i_{n}} U\left(a_{i_{1}} \cdots a_{i_{n-1}} a_{i_{n}} t\right)\right] \\
& \geq \sum_{i_{1}=1}^{k} \cdots \sum_{i_{n-1}=1}^{k} p_{i_{1}} \cdots p_{i_{n-1}}\left[\sum_{i_{n}=1}^{k} p_{i_{n}} U\left(a_{i_{1}} \cdots a_{i_{n-1}} t\right)\right] \\
& =\sum_{i_{1}=1}^{k} \cdots \sum_{i_{n-1}=1}^{k} p_{i_{1}} \cdots p_{i_{n-1}} U\left(a_{i_{1}} \cdots a_{i_{n-1}} t\right)=\mathrm{P}\left(X_{n} \leq t\right) .
\end{aligned}
$$

Thus, $X_{n} \geq_{\text {st }} X_{n+1}$. By a similar argument, from (9) we can prove that $Y_{n} \leq_{\text {st }} Y_{n+1}$. This completes the proof of Theorem 1 .

Theorem 1 shows that, under the additional Assumptions 1-3, the model is a monotone process model for a multistate deteriorating system. However, under Assumption $3^{\prime}$, instead of Theorem 1, we have the following result.

Theorem 2. Under Assumption $3^{\prime}$, for $n=1,2, \ldots$, we have $X_{n} \leq_{\mathrm{st}} X_{n+1}$ and $Y_{n} \geq_{\mathrm{st}} Y_{n+1}$.

The proof is similar to that of Theorem 1 .

Therefore, according to Theorem 2, under the additional Assumptions 1, 2, and 3', our model is a monotone process model for a multistate improving system.

\section{Long-run average cost per unit time}

In this section, we shall derive results based on the probability structure of the model and the additional Assumptions 1 and 2 only. To derive the long-run average cost per unit time, we should first evaluate the expected values of $X_{n}$ and $Y_{n}$. To do this, let $\int_{0}^{\infty} t \mathrm{~d} U(t)=\lambda$ and $\int_{0}^{\infty} t \mathrm{~d} V(t)=\mu$. Then, from (8), we have

$$
\begin{aligned}
\mathrm{E}\left(X_{n}\right) & =\sum_{\left\{j_{1}, \ldots, j_{k} \in \mathbb{Z}^{+}: \sum_{i=1}^{k} j_{i}=n-1\right\}} \frac{(n-1) !}{j_{1} ! \cdots j_{k} !} p_{1}^{j_{1}} \cdots p_{k}^{j_{k}} \int_{0}^{\infty} t \mathrm{~d} U\left(a_{1}^{j_{1}} \cdots a_{k}^{j_{k}} t\right) \\
& =\sum_{\left\{j_{1}, \ldots, j_{k} \in \mathbb{Z}^{+}: \sum_{i=1}^{k} j_{i}=n-1\right\}} \frac{(n-1) !}{j_{1} ! \cdots j_{k} !}\left(\frac{p_{1}}{a_{1}}\right)^{j_{1}} \cdots\left(\frac{p_{k}}{a_{k}}\right)^{j_{k}} \lambda \\
& =\lambda\left(\frac{p_{1}}{a_{1}}+\cdots+\frac{p_{k}}{a_{k}}\right)^{n-1}=\frac{\lambda}{a^{n-1}}
\end{aligned}
$$


where $a^{-1}=\sum_{i=1}^{k} p_{i} / a_{i}$. Similarly, from (10), we have

$$
\mathrm{E}\left(Y_{n}\right)=\mu\left(\frac{q_{1}}{b_{1}}+\cdots+\frac{q_{\ell}}{b_{\ell}}\right)^{n}=\frac{\mu}{b^{n}},
$$

where $b^{-1}=\sum_{i=1}^{\ell} q_{i} / b_{i}$.

Second, we should calculate the expected reward earned after the $(n-1)$ th repair and the expected repair cost incurred after the $n$th failure. For this purpose, define $R_{n}$, the reward rate after the $(n-1)$ th repair, as

$$
R_{n}=r_{i} \quad \text { if } S\left(t_{n-1}\right)=i, i=1, \ldots, k .
$$

Because $S\left(t_{0}\right)=1$, we have $R_{1}=r_{1}$ and, so, the expected reward after installation or one replacement is given by

$$
\mathrm{E}\left(R_{1} X_{1}\right)=\mathrm{E}\left(r_{1} X_{1}\right)=r_{1} \lambda .
$$

In general, for $n \geq 2$, the expected reward after the $(n-1)$ th repair is

$$
\begin{aligned}
\mathrm{E}\left(R_{n} X_{n}\right)= & \sum_{i_{1}=1}^{k} \cdots \sum_{i_{n-1}=1}^{k} \mathrm{E}\left(R_{n} X_{n} \mid S\left(t_{1}\right)=i_{1}, \ldots, S\left(t_{n-1}\right)=i_{n-1}\right) \\
& \times \mathrm{P}\left(S\left(t_{1}\right)=i_{1}, \ldots, S\left(t_{n-1}\right)=i_{n-1}\right) \\
= & \sum_{i_{1}=1}^{k} \cdots \sum_{i_{n-1}=1}^{k} p_{i_{1}} \cdots p_{i_{n-1}} \int_{0}^{\infty} r_{i_{n-1}} t \mathrm{~d} U\left(a_{i_{1}} \cdots a_{i_{n-1}} t\right) \\
= & \left(\sum_{i_{1}=1}^{k} \cdots \sum_{i_{n-2}=1}^{k} \frac{p_{i_{1}} \cdots p_{i_{n-2}}}{a_{i_{1}} \cdots a_{i_{n-2}}}\right)\left(\sum_{i_{n-1}=1}^{k} \frac{r_{i_{n-1}} p_{i_{n-1}}}{a_{i_{n-1}}}\right) \lambda \\
= & r \lambda\left(\frac{p_{1}}{a_{1}}+\cdots+\frac{p_{k}}{a_{k}}\right)^{n-2}=\frac{r \lambda}{a^{n-2}},
\end{aligned}
$$

where

$$
r=\sum_{i=1}^{k} \frac{r_{i} p_{i}}{a_{i}}
$$

To evaluate the expected repair cost incurred after the $n$th failure, define $C_{n}$, the repair-cost rate after the $n$th failure, as

$$
C_{n}=c_{i} \quad \text { if } S\left(s_{n}\right)=k+i, i=1, \ldots, \ell .
$$

By a calculation similar to that of $\mathrm{E}\left(R_{n} X_{n}\right)$, the expected repair cost after the $n$th failure is found to be

$$
\mathrm{E}\left(C_{n} Y_{n}\right)=c \mu\left(\frac{q_{1}}{b_{1}}+\cdots+\frac{q_{\ell}}{b_{\ell}}\right)^{n-1}=\frac{c \mu}{b^{n-1}},
$$

where

$$
c=\sum_{i=1}^{\ell} \frac{c_{i} q_{i}}{b_{i}}
$$


Now suppose that a replacement policy $N$ is adopted. We say that a cycle is completed if a replacement is completed; in other words, a cycle is the time interval between the installation and the first replacement or between two successive replacements. Thus, the successive cycles, and the costs incurred in each one, form a renewal reward process (see, e.g. Ross (1996)). Let $\tau=\mathrm{E}(Z)$ be the expected replacement time. Then, by applying a standard result on renewal reward processes, the long-run average cost per unit time (or, simply, the average cost) is given by

$$
\begin{aligned}
C(N) & =\frac{\text { expected cost incurred in a cycle }}{\text { expected length of a cycle }} \\
& =\frac{\mathrm{E}\left(\sum_{n=1}^{N-1} C_{n} Y_{n}+R+c_{p} Z-\sum_{n=1}^{N} R_{n} X_{n}\right)}{\mathrm{E}\left(\sum_{n=1}^{N} X_{n}+\sum_{n=1}^{N-1} Y_{n}+Z\right)} \\
& =\frac{c \mu \sum_{n=1}^{N-1}\left(1 / b^{n-1}\right)+R+c_{p} \tau-\left[r_{1} \lambda+r \lambda \sum_{n=2}^{N}\left(1 / a^{n-2}\right)\right]}{\lambda \sum_{n=1}^{N}\left(1 / a^{n-1}\right)+\mu \sum_{n=1}^{N-1}\left(1 / b^{n}\right)+\tau} \\
& =\frac{c \mu \sum_{n=1}^{N-1}\left(1 / b^{n-1}\right)-r \lambda \sum_{n=1}^{N}\left(1 / a^{n-2}\right)+R^{\prime}}{\lambda \sum_{n=1}^{N}\left(1 / a^{n-1}\right)+\mu \sum_{n=1}^{N-1}\left(1 / b^{n}\right)+\tau}
\end{aligned}
$$

or

$$
C(N)=\frac{b c(\mu / b) \sum_{n=1}^{N-1}\left(1 / b^{n-1}\right)-\operatorname{ar} \lambda \sum_{n=1}^{N}\left(1 / a^{n-1}\right)+R^{\prime}}{\lambda \sum_{n=1}^{N}\left(1 / a^{n-1}\right)+(\mu / b) \sum_{n=1}^{N-1}\left(1 / b^{n-1}\right)+\tau},
$$

where

$$
R^{\prime}=R+c_{p} \tau-\left(r_{1}-a r\right) \lambda
$$

Clearly, $a$ and $b$ are two important constants in (13) and (14). To understand the meaning of $a$ and $b$ better, we now introduce the harmonic mean of a random variable.

Definition 3. Given a random variable $X$ with $\mathrm{E}(1 / X) \neq 0, m_{\mathrm{H}}=[\mathrm{E}(1 / X)]^{-1}$ is the harmonic mean of $X$.

The harmonic mean has the following properties.

1. If $X$ is a discrete, uniformly distributed random variable such that $X=x_{i}$ with probability $1 / n, i=1, \ldots, n$, then the harmonic mean $m_{\mathrm{H}}=n / \sum_{i=1}^{n}\left(1 / x_{i}\right)$ is the harmonic mean of the numbers $x_{1}, \ldots, x_{n}$.

2. If $0<\alpha \leq X \leq \beta$ then $\alpha \leq m_{\mathrm{H}} \leq \beta$.

3. If $X$ is nonnegative with $\mathrm{E}(X)>0$ then $m_{\mathrm{H}} \leq \mathrm{E}(X)$. This is because $h(x)=1 / x$ is a convex function, from which the result follows by the Jensen inequality.

Thus, in our model, $a$ is the harmonic mean of random variable $A$ with $\mathrm{P}\left(A=a_{i}\right)=p_{i}$, $i=1, \ldots, k$, and $b$ is the harmonic mean of random variable $B$ with $\mathrm{P}\left(B=b_{i}\right)=q_{i}$, $i=1, \ldots, \ell$. For this reason, we may call $a$ and $b$ the harmonic means of $a_{1}, \ldots, a_{k}$ and $b_{1}, \ldots, b_{\ell}$, respectively.

Our problem now is to determine an optimal replacement policy for minimizing the average $\operatorname{cost} C(N)$. To this end, we first observe from (14) that

$$
C(N)=D(N)-a r
$$


where

$$
D(N)=\frac{(b c+a r)(\mu / b) \sum_{n=1}^{N-1}\left(1 / b^{n-1}\right)+R^{\prime}+\operatorname{ar} \tau}{\lambda \sum_{n=1}^{N}\left(1 / a^{n-1}\right)+(\mu / b) \sum_{n=1}^{N-1}\left(1 / b^{n-1}\right)+\tau} .
$$

Obviously, the problem of minimizing $C(N)$ is equivalent to the problem of minimizing $D(N)$. Therefore, consider

$$
\begin{aligned}
D(N+1)-D(N) & \left\{(b c+a r) \mu\left[\lambda\left(\sum_{n=1}^{N} a^{n}-\sum_{n=1}^{N-1} b^{n}\right)+\tau a^{N}\right]-\left(R^{\prime}+a r \tau\right)\left[\lambda b^{N}+\mu a^{N}\right]\right\} \\
& \times\left\{a^{N} b^{N}\left[\lambda \sum_{n=1}^{N} \frac{1}{a^{n-1}}+\frac{\mu}{b} \sum_{n=1}^{N-1} \frac{1}{b^{n-1}}+\tau\right] \times\left[\lambda \sum_{n=1}^{N+1} \frac{1}{a^{n-1}}+\frac{\mu}{b} \sum_{n=1}^{N} \frac{1}{b^{n-1}}+\tau\right]\right\}^{-1} .
\end{aligned}
$$

For $R^{\prime}+\operatorname{ar} \tau \neq 0$, we define an auxiliary function

$$
g(N)=\frac{(b c+a r) \mu\left[\lambda\left(\sum_{n=1}^{N} a^{n}-\sum_{n=1}^{N-1} b^{n}\right)+\tau a^{N}\right]}{\left(R^{\prime}+\operatorname{ar} \tau\right)\left[\lambda b^{N}+\mu a^{N}\right]}
$$

while, for $R^{\prime}+\operatorname{ar} \tau=0$, we define

$$
g_{0}(N)=(b c+a r) \mu\left[\lambda\left(\sum_{n=1}^{N} a^{n}-\sum_{n=1}^{N-1} b^{n}\right)+\tau a^{N}\right] .
$$

As the denominator of $D(N+1)-D(N)$ (i.e. the quantity in braces in (16)) is always positive, it is clear that the sign of $D(N+1)-D(N)$ is the same as that of its numerator. Consequently, we have the following lemma.

Lemma 1. 1. If $R^{\prime}+\operatorname{ar} \tau>0$ then

$$
\begin{aligned}
& D(N+1)>D(N) \Leftrightarrow g(N)>1, \\
& D(N+1)=D(N) \Leftrightarrow g(N)=1, \\
& D(N+1)<D(N) \Leftrightarrow g(N)<1 .
\end{aligned}
$$

2. If $R^{\prime}+\operatorname{ar} \tau<0$ then

$$
\begin{aligned}
& D(N+1)>D(N) \Leftrightarrow g(N)<1, \\
& D(N+1)=D(N) \Leftrightarrow g(N)=1, \\
& D(N+1)<D(N) \Leftrightarrow g(N)>1 .
\end{aligned}
$$

3. If $R^{\prime}+\operatorname{ar} \tau=0$ then

$$
\begin{aligned}
& D(N+1)>D(N) \Leftrightarrow g_{0}(N)>0, \\
& D(N+1)=D(N) \Leftrightarrow g_{0}(N)=0, \\
& D(N+1)<D(N) \Leftrightarrow g_{0}(N)<0 .
\end{aligned}
$$


Lemma 1 shows that the monotonicity of $D(N)$ can be determined by the value of either $g(N)$ or $g_{0}(N)$.

Let $h(N)=\lambda b^{N}+\mu a^{N}$. Then, for $R^{\prime}+\operatorname{ar} \tau \neq 0$, it follows from (17) that

$$
\begin{aligned}
g(N+1)-g(N)= & \frac{(b c+a r) \mu}{\left(R^{\prime}+a r \tau\right) h(N) h(N+1)} \\
& \times\left\{\lambda^{2} b^{N}(1-b) \sum_{n=1}^{N} a^{n}+\lambda^{2} b^{N}\left(a^{N+1}-b\right)+\lambda \mu a^{N}\left(a-b^{N}\right)\right. \\
& \left.+\lambda \mu a^{N}(a-1) \sum_{n=1}^{N-1} b^{n}+\lambda \tau a^{N} b^{N}(a-b)\right\} .
\end{aligned}
$$

For $R^{\prime}+\operatorname{ar} \tau=0$, it follows from (18) that

$$
g_{0}(N+1)-g_{0}(N)=(b c+a r) \mu\left\{\lambda\left(a^{N+1}-b^{N}\right)+\tau\left(a^{N+1}-a^{N}\right)\right\} .
$$

Note that, in this section, the results are all developed under the probability structure of the model and the additional Assumptions 1 and 2 only. Therefore, all the results, including Lemma 1, are true for our model of deteriorating systems and improving systems.

\section{The optimal policy for the deteriorating system}

Under Assumption 3, it follows from property 2 of the harmonic mean that

$$
1=a_{0} \leq a \leq a_{k-1} \quad \text { and } \quad b_{k-1} \leq b \leq b_{0}=1 .
$$

Consider a GP model for a two-state (up and down, say) deteriorating system. Assume that the successive operating times after repair $\left\{X_{n}^{\prime}, n=1,2, \ldots\right\}$ form a GP with ratio $a \geq 1$ and $\mathrm{E}\left(X_{1}^{\prime}\right)=\lambda$. Assume further that the consecutive repair times after failure $\left\{Y_{n}^{\prime}, n=1,2, \ldots\right\}$ constitute a GP with ratio $0<b \leq 1$ and $\mathrm{E}\left(Y_{1}^{\prime}\right)=\mu / b$. The replacement time is still $Z$, with $\mathrm{E}(Z)=\tau$. The reward rate is $a r$, the repair-cost rate is $b c$, and the replacement cost is $R^{\prime}$. Then, under policy $N$, an argument similar to that presented in the previous section will show that the average cost for the two-state system is exactly the same as that given by (14) (see Lam (1988a), (1988b), and (2003) for details). In other words, the monotone process model for a multistate system is equivalent to a GP model for the two-state system, in the sense that they will have the same average cost and, hence, the same optimal maintenance policy. In conclusion, we have proved the following theorem.

Theorem 3. The monotone process model for a one-component multistate deteriorating system is equivalent to a GP model for a one-component two-state deteriorating system in the sense that they will have the same average cost and the same optimal replacement policy. The successive operating times after repair $\left\{X_{n}^{\prime}, n=1,2, \ldots\right\}$ of the two-state system will form a GP with ratio $a \geq 1$ (the harmonic mean of $\left.a_{1}, \ldots, a_{k}\right)$ and $\mathrm{E}\left(X_{1}^{\prime}\right)=\lambda$. The consecutive repair times after failure $\left\{Y_{n}^{\prime}, n=1,2, \ldots\right\}$ will constitute a GP with ratio $0<b \leq 1$ (the harmonic mean of $\left.b_{1}, \ldots, b_{\ell}\right)$ and $\mathrm{E}\left(Y_{1}^{\prime}\right)=\mu / b$. The reward rate of the two-state system is ar with $r$ given by (11), the repair-cost rate is bc with $c$ given by (12), and the replacement cost is $R^{\prime}$, given by (15).

From (19), (20), and (21), the proof of the following lemma is straightforward. 
Lemma 2. 1. If $R^{\prime}+\operatorname{ar} \tau>0$ then $g(N)$ is nondecreasing in $N$.

2. If $R^{\prime}+\operatorname{ar} \tau<0$ then $g(N)$ is nonincreasing in $N$.

3. The function $g_{0}(N)$ is nondecreasing in $N$.

The combination of Lemmas 1 and 2 yields the following theorem.

Theorem 4. 1. For $R^{\prime}+$ ar $\tau>0$, an optimal replacement policy $N_{\mathrm{d}}^{*}$ for the multistate deteriorating system is given by

$$
N_{\mathrm{d}}^{*}=\min \{N \mid g(N) \geq 1\} .
$$

The optimal policy $N_{\mathrm{d}}^{*}$ is unique if and only if $g\left(N_{\mathrm{d}}^{*}\right)>1$.

2. For $R^{\prime}+$ ar $\tau<0$, an optimal replacement policy $N_{\mathrm{d}}^{*}$ for the multistate deteriorating system is given by

$$
N_{\mathrm{d}}^{*}=\min \{N \mid g(N) \leq 1\} .
$$

The optimal policy $N_{\mathrm{d}}^{*}$ is unique if and only if $g\left(N_{\mathrm{d}}^{*}\right)<1$.

3. For $R^{\prime}+\operatorname{ar} \tau=0$, an optimal replacement policy $N_{\mathrm{d}}^{*}$ for the multistate deteriorating system is given by

$$
N_{\mathrm{d}}^{*}=\min \left\{N \mid g_{0}(N) \geq 0\right\} .
$$

The optimal policy $N_{\mathrm{d}}^{*}$ is unique if and only if $g_{0}\left(N_{\mathrm{d}}^{*}\right)>0$.

In applications of Theorem 4, we should determine the value of $R^{\prime}+\operatorname{ar} \tau$ first. As an example, suppose that $R^{\prime}+\operatorname{ar} \tau>0$; we then determine $N_{\mathrm{d}}^{*}$ from (22), and we have

$$
g(N)<1 \Leftrightarrow N<N_{\mathrm{d}}^{*}
$$

and

$$
g(N) \geq 1 \Leftrightarrow N \geq N_{\mathrm{d}}^{*}
$$

Thus, $N_{\mathrm{d}}^{*}$ is the minimum integer satisfying (22). By Lemma 1, we have

$$
D(N)>D\left(N_{\mathrm{d}}^{*}\right) \quad \text { if } N<N_{\mathrm{d}}^{*}
$$

and

$$
D(N) \geq D\left(N_{\mathrm{d}}^{*}\right) \quad \text { if } N \geq N_{\mathrm{d}}^{*} .
$$

In other words, policy $N_{\mathrm{d}}^{*}$ is indeed an optimal replacement policy. Clearly, it is unique if and only if $g\left(N_{\mathrm{d}}^{*}\right)>1$.

Of course, Theorem 4 can be applied not only to a multistate deteriorating system but also to a two-state deteriorating system.

\section{The monotone process model for an improving system}

Now, under Assumption $3^{\prime}$, we have

$$
a_{k} \leq a \leq a_{1}=1 \text { and } 1=b_{1} \leq b \leq b_{\ell} .
$$

Also, the equalities in (23) do not all hold simultaneously.

As in the case of the multistate deteriorating system, we can consider a GP model for a twostate improving system. Consequently, we have the following theorem, which is analogous to Theorem 3. 
Theorem 5. The monotone process model for a one-component multistate improving system is equivalent to a GP model for a one-component two-state improving system in the sense that they have the same average cost and the same optimal replacement policy. The successive operating times after repair $\left\{X_{n}^{\prime}, n=1,2, \ldots\right\}$ of the two-state system will form a GP with ratio $0<a \leq 1$ (the harmonic mean of $a_{1}, \ldots, a_{k}$ ) and $\mathrm{E}\left(X_{1}^{\prime}\right)=\lambda$. The consecutive repair times after failure $\left\{Y_{n}^{\prime}, n=1,2, \ldots\right\}$ will constitute a GP with ratio $b \geq 1$ (the harmonic mean of $\left.b_{1}, \ldots, b_{\ell}\right)$ and $\mathrm{E}\left(Y_{1}^{\prime}\right)=\mu / b$. The reward rate of the two-state system is ar with $r$ given by (11), the repair-cost rate is bc with $c$ given by (12), and the replacement cost is $R^{\prime}$, given by (15).

Note that, for an improving system, the average cost due to the adoption of a replacement policy $N$ is also given by (14). Moreover, we can also determine an optimal replacement policy for the improving system on the basis of Lemma 1. To do so, recall that not all equalities in (23) will hold simultaneously. Then, from (19), (20), and (23), $g(N)$ and $g_{0}(N)$ are clearly strictly monotone. Thus in analogy with Lemma 2 , we have the following result.

Lemma 3. 1. If $R^{\prime}+\operatorname{ar} \tau>0$ then $g(N)$ is decreasing in $N$.

2. If $R^{\prime}+\operatorname{ar} \tau<0$ then $g(N)$ is increasing in $N$.

3. The function $g_{0}(N)$ is decreasing in $N$.

Now we are available to determine the optimal replacement policy for the multistate improving system.

Theorem 6. For the multistate improving system, if

$$
r_{1} \leq r_{i}, \quad i=2, \ldots, k,
$$

then $N_{\mathrm{i}}^{*}=\infty$ is the unique optimal replacement policy.

Proof. We shall prove Theorem 6 by considering three cases.

Case 1. For $R^{\prime}+\operatorname{ar} \tau>0, g(N)$ is decreasing, so let

$$
N_{+}=\min \{N \mid g(N) \leq 1\} .
$$

Thus, we have

$$
g(N)>1 \Leftrightarrow N<N_{+}
$$

and

$$
g(N) \leq 1 \Leftrightarrow N \geq N_{+} .
$$

By Lemma 1, it is easy to see that $C(N)$ and $D(N)$ are unimodal and will take their maxima at $N_{+}$. This implies that the minimum of $C(N)$ must be given by $\min \{C(1), C(\infty)\}$. Note, from (24), that $r_{1} \leq a r$. Then (14) yields

$$
C(1)=\frac{R+c_{p} \tau-r_{1} \lambda}{\lambda+\tau}>C(\infty)=-a r
$$

Consequently,

$$
\min C(N)=\min \{C(1), C(\infty)\}=C(\infty)=-a r .
$$

Therefore, $N_{\mathrm{i}}^{*}=\infty$ is the optimal replacement policy. The optimal policy is also unique because, from Lemma 3, $g(N)$ is strictly decreasing. 
Case 2. For $R^{\prime}+\operatorname{ar} \tau<0$, the proof is similar.

Case 3. For $R^{\prime}+\operatorname{ar} \tau=0$, because $g_{0}(N)$ is decreasing there exists an

$$
N_{0}=\min \left\{N \mid g_{0}(N) \leq 0\right\} .
$$

Thus, we have

$$
g_{0}(N)>0 \Leftrightarrow N<N_{0}
$$

and

$$
g_{0}(N) \leq 0 \Leftrightarrow N \geq N_{0} .
$$

An argument similar to the above shows that $C(N)$ and $D(N)$ will attain their maxima at $N_{0}$ and, consequently, that $N_{\mathrm{i}}^{*}=\infty$ is the unique optimal replacement policy.

This completes the proof.

Because the $k$ working states are arranged in increasing order (in the sense defined above) with state 1 the worst, the worse the working state is, the lower is the reward rate. Therefore, condition (24) is a natural and reasonable assumption. Consequently, for most practical multistate improving systems, Theorem 6 is applicable. As a result, we should continually repair the system as it ages, without ever replacing it.

Like Theorem 4, Theorem 6 can apply not only to a multistate improving system but also to a two-state improving system.

\section{Acknowledgements}

The author is grateful to the editor and referee for their comments and suggestions, which have much improved the presentation.

\section{References}

Lam, Y. (1988a). A note on the optimal replacement problem. Adv. Appl. Prob. 20, 479-482.

Lam, Y. (1988b). Geometric processes and replacement problem. Acta Math. Appl. Sinica 4, 366-377.

LAM, Y. (1991a). An optimal repairable replacement model for deteriorating systems. J. Appl. Prob. 28, 843-851.

LAM, Y. (1991b). Optimal policy for a general repair replacement model: average reward case. IMA J. Math. Appl. Business Industry 3, 117-129.

LAM, Y. (1992a). Nonparametric inference for geometric processes. Commun. Statist. Theory Meth. 21, $2083-2105$.

LAM, Y. (1992b). Optimal policy for a general repair replacement model: discounted reward case. Commun. Statist. Stoch. Models 8, 245-267.

LAM, Y. (1995). Calculating the rate of occurrence of failures for continuous-time Markov chains with application to a two-component parallel system. J. Operat. Res. Soc. 46, 528-536.

LAM, Y. (2003). A geometric process maintenance model. Southeast Asian Bull. Math. 27, 295-305.

LAM, Y. (2005). Geometric process. To appear in The Encyclopedia of Statistical Sciences, 2nd edn., eds N. Balakrishnan, C. Read, S. Kotz and B. Vidakovic, John Wiley, New York.

Lam, Y. AND Chan, S. K. (1998). Statistical inference for geometric processes with lognormal distribution. Comput. Statist. Data Anal. 27, 99-112.

LAM, Y. AND ZHANG, Y. L. (1996). Analysis of a two-component series system with a geometric process model. Naval Res. Logistics 43, 491-502.

LAM, Y., Zhang, Y. L. And Zheng, Y. H. (2002). A geometric process equivalent model for a multistate degenerative system. Europ. J. Operat. Res. 142, 21-29.

Lam, Y., Zhu, L. X., Chan, S. K. AND LiU, Q. (2004). Analysis of data from a series of events by a geometric process model. Acta Math. Appl. Sinica 20, 263-282.

Leung, F. K. N. AND LeE, Y. M. (1998). Using geometric processes to study maintenance problems for engines. Internat. J. Ind. Theory 5, 316-323.

Morimura, H. (1970). On some preventive maintenance policies for IFR. J. Operat. Res. Soc. Japan 12, 94-124.

PARK, K. S. (1979). Optimal number of minimal repairs before replacement. IEEE Trans. Reliab. 28, 137-140. 
Pérez-Ocón, R. And Torres-Castro, I. (2002). A reliability semi-Markov model involving geometric processes. Appl. Stoch. Models Business Industry 18, 157-170.

Ross, S. M. (1996). Stochastic Processes, 2nd edn. John Wiley, New York.

Shaked, M. And Shanthikumar, J. G. (1994). Stochastic Orders and Their Applications. Academic Press, San Diego, CA.

Sheu, S. H. (1999). Extended optimal replacement model for deteriorating systems. Europ. J. Operat. Res. 112, 503-516.

Stadje, W. and Zuckerman, D. (1990). Optimal strategies for some repair replacement models. Adv. Appl. Prob. 22, 641-656.

Zhang, Y. L. (1999). An optimal geometric process model for a cold standby repairable system. Reliab. Eng. Systems Safety 63, 107-110.

ZhanG, Y. L., YAM, R. C. M. And Zuo, M. J. (2002). Optimal replacement policy for a multistate repairable system. J. Operat. Res. Soc. 53, 336-341. 